\title{
Pulsed Photoelectric Coherent Manipulation and Detection of N-V Center Spins in Diamond
}

\author{
Michal Gulka, ${ }^{1,2}$ Emilie Bourgeois, ${ }^{1,3}$ Jaroslav Hruby, ${ }^{1}$ Petr Siyushev, ${ }^{4}$ Georg Wachter, ${ }^{5}$ Friedrich Aumayr, ${ }^{6}$ \\ Philip R. Hemmer, ${ }^{7}$ Adam Gali, ${ }^{8,9}$ Fedor Jelezko, ${ }^{4}$ Michael Trupke, ${ }^{5}$ and Milos Nesladek ${ }^{1,3, *}$ \\ ${ }^{1}$ Institute for Materials Research (IMO), Hasselt University, \\ Wetenschapspark 1, B-3590 Diepenbeek, Belgium \\ ${ }^{2}$ Faculty of Biomedical Engineering, Czech Technical University in Prague, \\ Sitna sq. 3105, 27201 Kladno, Czech Republic \\ ${ }^{3}$ IMOMEC division, IMEC, Wetenschapspark 1, B-3590 Diepenbeek, Belgium \\ ${ }^{4}$ Institute for Quantum Optics and IQST, Ulm University, \\ Albert-Einstein-Allee 11, D-89081 Ulm, Germany \\ ${ }^{5}$ Vienna Center for Quantum Science and Technology, Atominstitut, TU Wien, 1020 Vienna, Austria \\ ${ }^{6}$ Institute of Applied Physics, TU Wien, Wiedner Hauptstrasse 8-10, 1040 Vienna, Austria \\ ${ }^{7}$ Department of Electrical and Computer Engineering, Texas A\&M University, \\ College Station, Texas 77843, USA \\ ${ }^{8}$ Institute for Solid State Physics and Optics, Wigner Research Centre for Physics, \\ Hungarian Academy of Sciences, PO Box 49, H-1525 Budapest, Hungary \\ ${ }^{9}$ Department of Atomic Physics, Budapest University of Technology and Economics, \\ Budafoki út 8, H-1111 Budapest, Hungary
}

(Received 10 November 2016; revised manuscript received 17 February 2017; published 28 April 2017; corrected 8 June 2017)

\begin{abstract}
We demonstrate hybrid photoelectric pulse protocols for reading the spin states of nitrogen-vacancy $(\mathrm{N}-V)$ centers in diamond, compatible with coherent spin control and performed on shallow nitrogen-implanted electronic grade diamond. The measurements are carried out on spin ensembles from 1000 to just five $\mathrm{N}-V$ centers as a first step towards the fabrication of scalable photoelectric quantum chips. Specific microwave protocols are developed that suppress background photocurrent related to ionization of $\mathrm{N}_{S}{ }^{0}$ defects and provide a high contrast and SNR. The technique is demonstrated on Rabi and Ramsey sequences.
\end{abstract}

DOI: 10.1103/PhysRevApplied.7.044032

\section{INTRODUCTION}

Hybrid quantum detection approaches are considered very promising in the realization of scalable systems for quantum computation [1]. Here we demonstrate coherent manipulation and photoelectric readout of nitrogenvacancy $(\mathrm{N}-V)$ spin states in diamond as a basic building block for scalable miniaturized electric quantum devices [2]. The developed readout protocols are based on the $\mathrm{N}-\mathrm{V}$ center two-photon ionization. Further on, by providing microwave (MW) on-resonance-triggering for a lock-in amplification, the photocurrent induced by the photoionization of single substitutional nitrogen $\mathrm{N}_{S}{ }^{0}$ ( $P 1$ defect) is subtracted from the detected signal. The presented technique allows for high readout contrast - the offset only due to the electronic noise - and signal readout up to high frequencies of approximately $20 \mathrm{MHz}$. The high sensitivity of the proposed protocols allows us to read the spin state of approximately five $\mathrm{N}-V$ centers.

$\mathrm{N}-V$ solid-state qubits in diamond are among the most studied quantum systems today due to their record spincoherence time at room temperature [3] and are used for quantum sensing applications (e.g., magnetometry [4],

*Corresponding author. milos.nesladek@uhasselt.be thermometry [5], or single-spin nano-magnetic-resonance imaging [6]). The optically detected magnetic resonance (ODMR) method [7] used as a major technique for $\mathrm{N}-V$ center readout in diamond has important limitations in the scalability and does not provide integration with electronic chip. Recently, we have introduced the photoelectrically detected magnetic resonance (PDMR) technique [8] for reading out the electron spin state of negatively charged $\mathrm{N}-\mathrm{V}$ center in diamond. This method provides a vast enhancement of over 2.5 orders of magnitude in detection rates compared to ODMR measured in similar experimental conditions (see Ref. [9] for details). The technique is potentially applicable to other materials such as $\mathrm{SiC}$ [10], $\mathrm{GaN}$, or AlN [11]. Although we have demonstrated the principle of this method, it was used only for continuouswave (CW) readout of the $|0\rangle$ and $| \pm 1\rangle$ spin states of $\mathrm{N}-V$ ensembles in lab-grown chemical vapor deposition (CVD) or high pressure high temperature (HPHT) diamonds [8]. In order to use the advantages of the PDMR technique in quantum sensing and computational schemes, it is necessary to demonstrate that the photoelectric readout is compatible with the quantum control of individual $\mathrm{N}-V$ electron spins. The photoelectric detection of Rabi oscillations was demonstrated, for example, in two-level exciton systems in quantum dots [12,13] or in silicon [14-16]. Here, we perform coherent operations with PDMR on spin ensembles 
of just a few (approximately five) $\mathrm{N}-V$ centers by designing specific readout sequences. We demonstrate these protocols by performing Rabi and Ramsey measurements on shallow $\mathrm{N}-V$ ensembles implanted in electronic grade diamond that is - unlike bulk crystals - a material suitable for future quantum devices. The proposed method leads to a significant suppression of background photocurrents, which is one of the main limiting factors in achieving PDMR on single $\mathrm{N}-V$ sites $[8,17]$ and considerably increased signal-to-noise ratio (SNR) compared to CW PDMR measurements [8,9]. In this way, we can experimentally verify the SNRs that were previously only theoretically predicted [18].

\section{EXPERIMENTAL SETUP AND PRINCIPLE}

Figure 1(a) depicts the hybrid photoelectric quantum chip used in our measurements. Type-IIa single-crystal electronic grade diamond is implanted with $8-\mathrm{keV}^{14} \mathrm{~N}^{4+}$ ions and annealed at $900{ }^{\circ} \mathrm{C}$ to create ensembles of shallow $\mathrm{N}-\mathrm{V}$ centers (depth $12 \pm 4 \mathrm{~nm}$ ). The implantation fluence is varied to create a total of five regions with different $\mathrm{N}-V$ densities (from approximately 15 to $10^{4} \mu \mathrm{m}^{-2}$ ). The sample is equipped with coplanar Ti-Au electrodes with a $50-\mu \mathrm{m}$ gap and mounted on a circuit board to enable MW excitation and photocurrent readout. The MW is provided using two printed antennas [19]. Green laser illumination induces the two-photon ionization of $\mathrm{N}-V$ centers from the $|0\rangle$ and $| \pm 1\rangle$ ${ }^{3} A_{2} \mathrm{~N}-V$ ground-state (GS) spin manifolds to the conduction band (CB) [Fig. 1(b)] [20,21]. A dc electric field is applied in between the electrodes, and the photocurrent is read out by a lock-in amplification after sensitive preamplification (see Ref. [22] for more details on the experimental setup).

The magnetic resonance (MR) contrast in the detected photocurrent is obtained under resonant microwave excitation $(2.87 \mathrm{GHz})$ inducing transitions from the $|0\rangle$ to the $| \pm 1\rangle$ spin sublevels of the N- $V$ ground state [23]. Electrons photoexcited from the $| \pm 1\rangle$ GS manifold have a nonzero probability of undergoing shelving transitions from the $\mathrm{N}-V$ excited state to the metastable ${ }^{1} E$ state [24-26]. During the time in which the $| \pm 1\rangle$ spin electron is stored in the metastable singlet state (approximately 220-ns lifetime) $[8,27,28]$, it is protected from photoionization. This electron shelving leads to a reduction of the overall photocurrent and, thus, to a minimum in the photocurrent signal at the resonant MW frequency [8]. So far, the photocurrent signal from $\mathrm{N}-V$ centers is read out either by the lock-in technique referenced to the laser-pulse frequency $[8,9]$ or by detecting the dc photocurrent after implementing a laser readout pulse [18]. Both proposed methods suffer from a background photocurrent induced by the photoionization of other defects such as substitutional nitrogen $\left(\mathrm{N}_{S}{ }^{0}\right)$ [9], which reduces the detected MR contrast, preventing the application of PDMR for single $\mathrm{N}-V$ detection. Here we perform PDMR readout using pulse sequences and referencing the lock-in signal to the MW pulses. In this way, we detect only the proportion of the photocurrent affected by the MW field, i.e., variations in the photocurrent signal related to $|0\rangle \leftrightarrow| \pm 1\rangle$ transitions. Therefore, we obtain a resonance spectrum with a positive contrast defined as $\left(S_{\max }-S_{\min }\right) /\left(S_{\max }+S_{\min }\right)$, where $S$ is the ac signal detected by the lock-in amplifier, and its maximum is equivalent to approximately $30 \%$ (or less) of the total dc off-resonant $\mathrm{N}-V$ photocurrent. Because the ac off-resonant photocurrent is zero, the detection contrast between off- and on-resonant MW frequencies is approximately $100 \%$ and is reduced only by the detected noise. As an additional advantage, the background signal from defects other than $\mathrm{N}-V$ is filtered out when scanning the MW frequency around the N-V resonant line. The use of MWtriggering sequences gives significantly lower electronic noise that is identified to originate from the coupling of MW irradiation to photocurrent readout circuits. To quantify the benefit of the MW-triggering scheme, we measure CW PDMR spectra at the same position using both approaches (a)

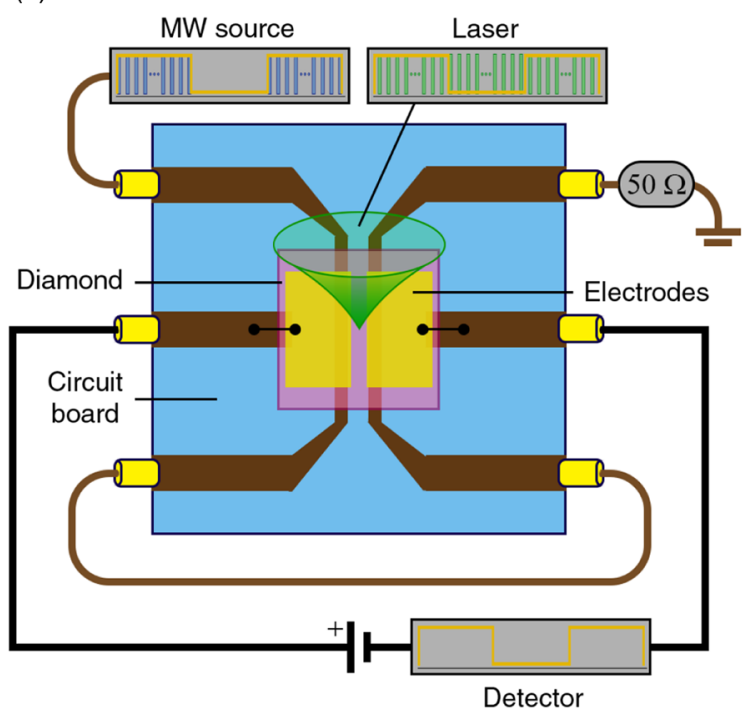

(b)
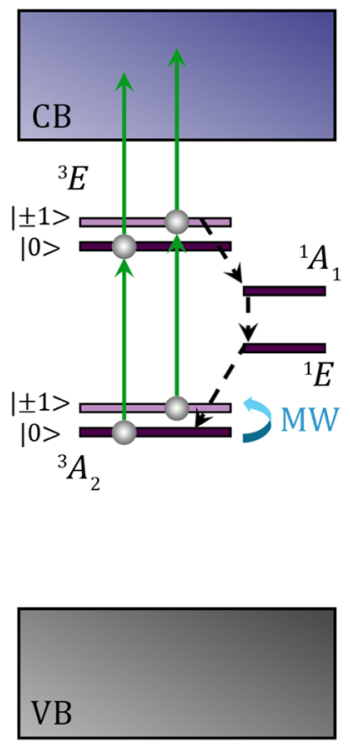

FIG. 1. (a) Schematics of the setup used for pulsed PDMR, showing a type-IIa nitrogenimplanted diamond mounted on the circuit board. (b) Simplified electronic energy-level scheme of the N-V center showing twophoton ionization of the electron from the GS to the CB. Spin-selective decay through metastable ${ }^{1} E$ state enables the PDMR (MW, microwave; $\mathrm{VB}$, valence band; $\mathrm{CB}$, conduction band). 

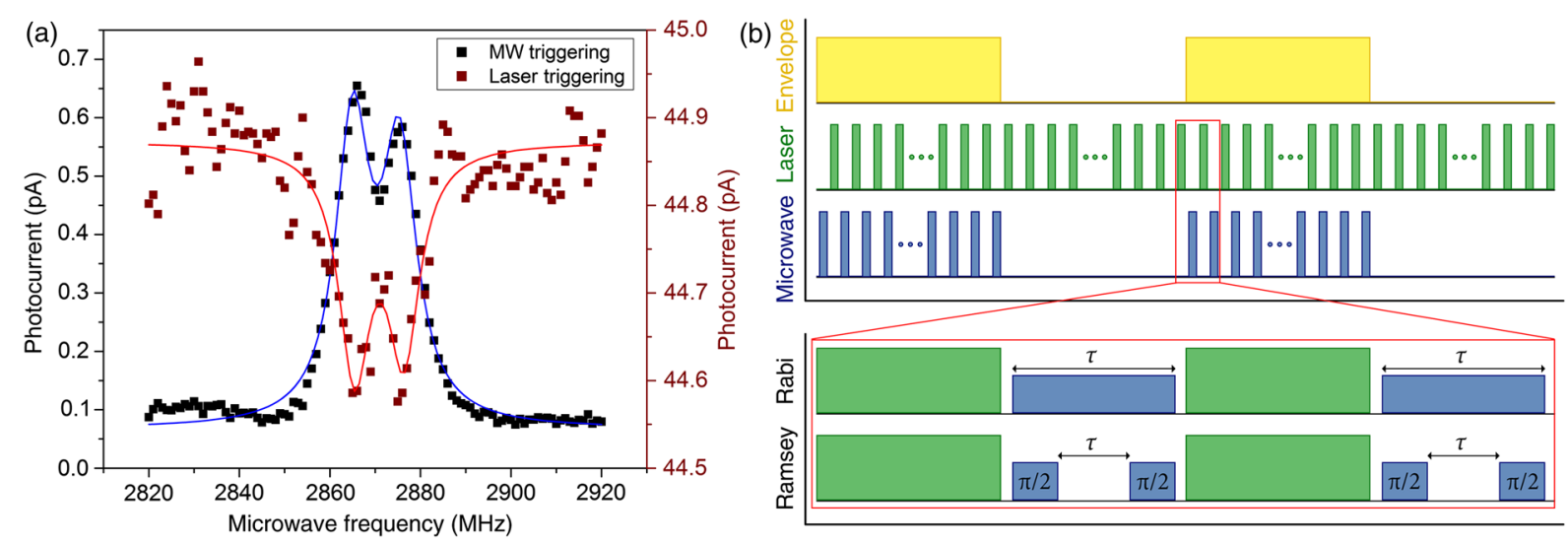

FIG. 2. (a) Comparison of the laser- (dark red dots) and MW- (black dots) triggering CW PDMR method in the case of a fast measurement (single PDMR scan). Data were measured on a N- $V$ ensemble using similar experimental conditions and fitted. (b) PDMR pulse trains that encode high-frequency pulse sequences (Rabi, Ramsey) into a low-frequency envelope used as a reference for lock-in detection.

(lock-in amplifier referenced to the laser and MW pulse frequency, with similar experimental conditions), and we compare the SNRs for both cases. The photocurrent signal $S$ is determined from a fit of the PDMR spectrum. The noise $N$ is calculated as the standard deviation of the off-resonant (blank) photocurrent signal. At the highly implanted region of the sample (corresponding to approximately $1000 \mathrm{~N}-V \mathrm{~s}$ in focus of the objective), the MW triggering yields a 7.4 times improvement in the SNR. The illustrative spectra are shown in Fig. 2(a). The vast improvement in the SNR is visible even for a very fast measurement.

To achieve coherent manipulation of spins with photoelectric readout, we encode single-pulse sequences into a low-frequency envelope [Fig. 2(b)]. A continuous series of consecutive laser pulses is used in which each pulse serves for the spin initialization and spin-state readout. Bursts of spin manipulation MW pulses time shifted with respect to laser pulses are added to encode any arbitrary sequence. A MW trigger pulse marks the start of the pulse burst during which the low-frequency-modulated photocurrent signal is measured. In the off period of the duty cycle, a train of spin-polarization laser pulses maintains the occupation of the $|0\rangle$ state. We explore envelope frequencies from $30 \mathrm{~Hz}$ up to $1 \mathrm{kHz}$, tuned to obtain the highest SNR. In principle, this method enables us to encode any pulse sequence into the low-frequency envelope. Here we demonstrate this technique on Rabi and Ramsey measurements, which are key sequences for quantum measurements [29].

\section{EXPERIMENTAL RESULTS AND DISCUSSION}

Rabi measurements (see Fig. 3) are performed first in the region of the sample with approximately $1000 \mathrm{~N}-V$ centers in the focus of the objective (region R1 [22]). Static magnetic field is applied perpendicularly to the $\langle 100\rangle$ diamond plane to split the $| \pm 1\rangle$ spin levels. We then vary the duration of resonant MW pulses at constant power [see Fig. 2(b)] to determine the duration of the $\pi$ pulse.
As expected, the Rabi frequencies calculated [22] from the fit [30] of these measurements depend linearly on the square root of the MW power (Fig. 3 inset). The presented measurement proves the possibility of coherent spin readout using the MW-triggered PDMR envelope pulse scheme. The measured Rabi oscillations for MW peak power of $3.2 \mathrm{~W}$ are plotted in Fig. 3, yielding a Rabi decay time of approximately $490 \mathrm{~ns}$ on the sample area with the highest ${ }^{14} \mathrm{~N}^{4+}$ ion implantation fluency.

To show the applicability of the designed PDMR protocols, we demonstrate functional Ramsey fringe pulse sequences [Fig. 2(b)] employed to characterize the $\mathrm{N}-V$ center free-spin dynamics. The measurement is performed

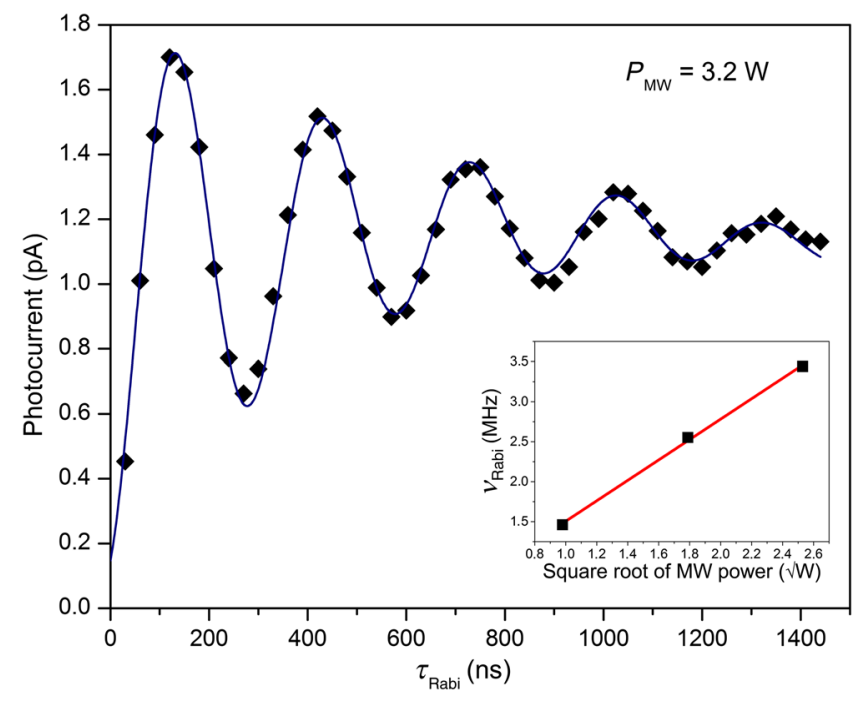

FIG. 3. Photoelectric detection of Rabi oscillations using the envelope technique with MW triggering. The black scatter points are the experimental data for MW power of $3.2 \mathrm{~W}$, and the blue line is fitted assuming an exponential damping. Inset shows the dependence of the frequency of Rabi oscillations with respect to the square root of the applied microwave power (red line is the result of the linear fit). 
at the same position as the Rabi experiment. By varying the free-precession time $\tau_{\text {Ram }}$, we observe a decay curve consisting of the superposition of three cosine functions with different frequencies $\delta_{i}$ (for conditions, see Ref. [22]). Each frequency corresponds to the detuning of the applied MW frequency from one of the hyperfine ${ }^{14} \mathrm{~N}$ transitions such that $\delta_{1}=\nu_{\text {Ram }}-2.2 \mathrm{MHz}, \delta_{2}=\nu_{\mathrm{Ram}}$, and $\delta_{3}=\nu_{\mathrm{Ram}}+2.2 \mathrm{MHz}$ [31,32], where $\nu_{\text {Ram }}$ is the Ramsey frequency. The measurement is performed for different detunings $\delta_{2}(4,8,12,16$, $20 \mathrm{MHz}$ ) from the central resonant frequency. The results are shown in Fig. 4. As expected, we observe a linear dependence of the Ramsey frequency to the detuning values (Fig. 4 inset). The average dephasing time $T_{2}^{*}$ for all measurements is approximately $41 \mathrm{~ns}$ using single exponential decay fitting [33] or approximately $66 \mathrm{~ns}$ using Gaussian decay fitting of the data [34]. This short value reflects the decoherent interactions of $\mathrm{N}-V$ ensembles on the sample area overimplanted with ${ }^{14} \mathrm{~N}^{4+}$ ions (region R1 [22]). The main source of dephasing is the bath of paramagnetic substitutional nitrogen impurities $\left(\mathrm{N}_{S}{ }^{0}\right.$ centers), although ${ }^{14} \mathrm{~N}$ nuclear spins can also contribute $[27,35,36]$. Despite the very fast decay, our technique enables us to read out Ramsey fringes at high frequency of $20 \mathrm{MHz}$ with a very high contrast, confirming the ability of MW-referenced PDMR to measure samples with very short $T_{2}^{*}$ times with high SNR. By the proposed technique, Hahn echo or more complex sequences such as the Carr-Purcell-Meiboom-Gill pulse train can be encoded into the envelope carrier. The base value of the MW-triggered photocurrent is inversely proportional to the detuning frequency, reflecting the occupation of spin states integrated over the statistical weight of the $|0\rangle$ and $|-1\rangle$ manifolds.

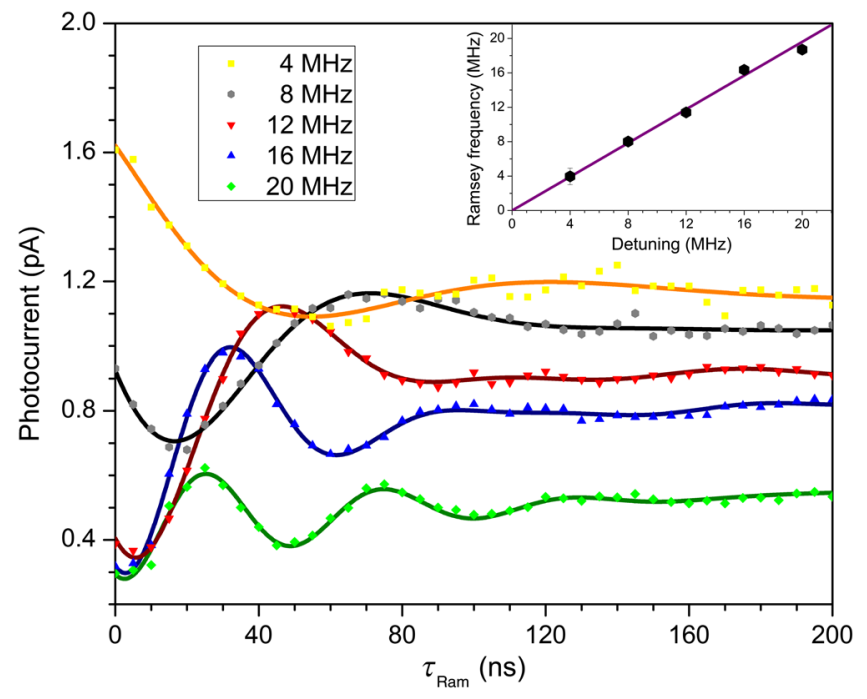

FIG. 4. Photoelectric detection of Ramsey fringes for different detuning of MW excitation frequency from the $\mathrm{N}-V$ resonant frequency. The scatter points are the experimental data fitted considering a single exponential decay (continuous lines). Inset shows the linear dependence of Ramsey frequency to the MW detuning (purple line is the result of the linear fit).
An important milestone for the PDMR technique is to reach coherent manipulation of single $\mathrm{N}-V$ spins. While our recent studies [8,9] and other published work [18] demonstrate PDMR on N- $V$ ensembles, the photoelectric readout of single spins is not achieved yet. To downscale the number of $\mathrm{N}-V$ centers, we use the $\mathrm{N}$-implanted electronic grade diamond containing five regions of descending $\mathrm{N}-V$ densities corresponding to ensembles from 1000 to approximately five $\mathrm{N}-V$ centers in focus of the objective. The number of $\mathrm{N}-V$ centers is calibrated from photoluminescence counts of a single $\mathrm{N}-V$ obtained under the same conditions.

First, we measure the total photocurrent signal upon downscaling the $\mathrm{N}-V$ ensembles [see Fig. 5(a) inset]. For this, we use the lock-in technique referenced to a pulsed laser without the MW field applied. These data can be fitted using a sublinear function. We speculate that this is due to a reduction of recombination lifetime in the highly implanted region. From the fit, we experimentally determine the total photocurrent resulting from the ionization of a single $\mathrm{N}-V$ to be approximately $1.0 \mathrm{pA}$ for an electric field
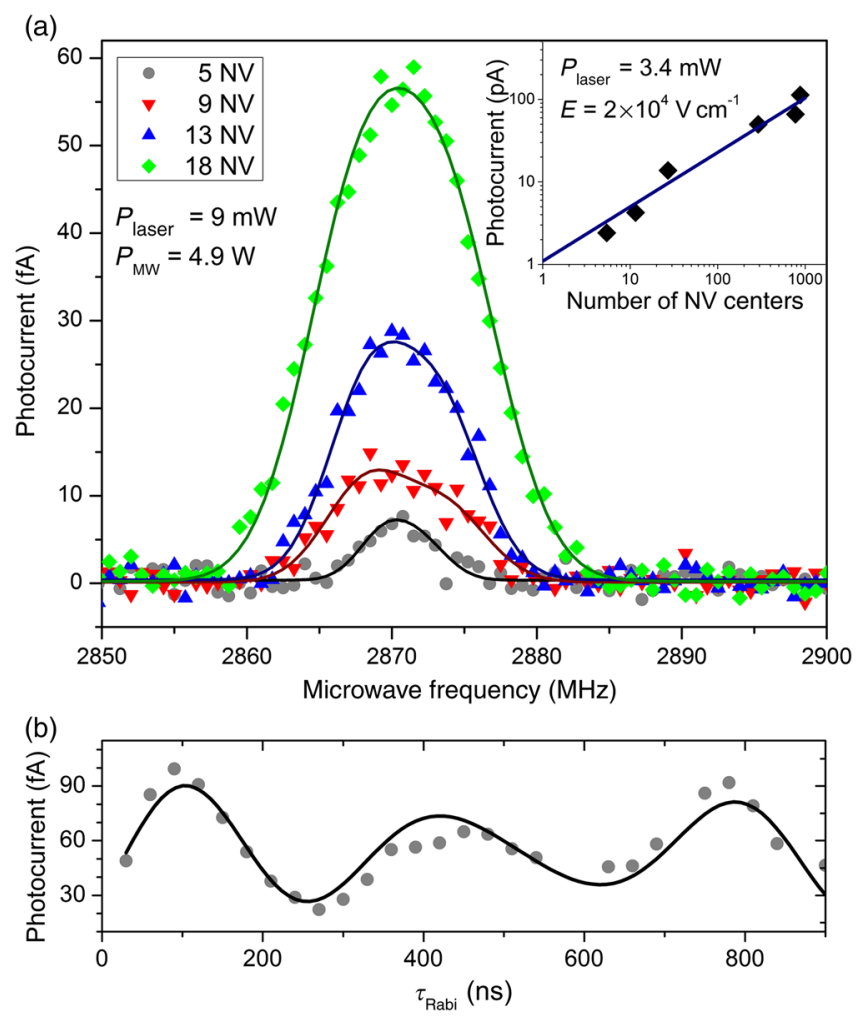

FIG. 5. (a) Photocurrent spectra measured on small spin ensembles down to five $\mathrm{N}-V$ s using a sequence consisting of a short laser pulse followed by a MW $\pi$ pulse. The experimental data are fitted with two Gaussian functions. For comparison, the inset shows the total photocurrent from different numbers of $\mathrm{N}-V$ centers measured with only laser excitation (blue line is the result of the fit). (b) Rabi measurement on five $\mathrm{N}-V$ centers at MW power of $3 \mathrm{~W}$. The fit of the data takes into account the beating originating from the coupling to the nitrogen nuclear spins with frequency of 2.2 MHz, similar to the fitting of Ramsey data in Fig. 4. 
of $2 \times 10^{4} \mathrm{~V} \mathrm{~cm}^{-1}$ similar to predictions presented in Refs. $[8,18]$. However, the measured photocurrent is a sum of the $\mathrm{N}-V$-related photocurrent and of the background signal originating mainly from single substitutional nitrogen $\mathrm{N}_{S}{ }^{0}$ ionization. We estimate that at these power conditions, approximately $75 \%$ of the signal comes from two-photon ionization of the $\mathrm{N}-V$ centers [9]. Thus, we experimentally verify the predictions of the photocurrent value expected for single $\mathrm{N}-V$ centers in given electric field conditions by measurements on a small number of $\mathrm{N}-V$ centers (approximately five) downscaled by at least approximately $10^{4}$ times compared to previous measurements [18].

Figure 5(a) shows pulse PDMR spectra obtained for decreasing numbers of $\mathrm{N}-V$ centers in the focus of the objective. The data are fitted using a two-peak Gaussian function [36] averaging over the central and hyperfine MR lines and a noise background offset is subtracted from the spectra. The estimated value of the single $\mathrm{N}-V$ photocurrent at resonance frequency averaged from all four measurements is approximately $2 \mathrm{fA}$. This value is approximately 50 times lower than the photocurrent expected for a single $\mathrm{N}-V$ in the used conditions based on CW light-triggered measurements (see above) and considering a CW PDMR contrast of approximately 10\% [9]. This decrease is attributed to several factors such as the use of the duty cycle (resulting in a reduced effective MW and laser power) and the loss of the contrast during the laser readout pulse (since the laser pulse is used for both readout and initialization of the $\mathrm{N}-V \mathrm{~s})$. By using pulse PDMR protocols with MW triggering, i.e., without any contribution of background photocurrent originating from $\mathrm{N}_{S}{ }^{0}$ photoionization, we demonstrate Rabi measurement on the sample region with the lowest implantation density corresponding to five $\mathrm{N}-V \mathrm{~s}$ in the focus [Fig. 5(b)]. The presented results show the applicability of the scheme for measurements of just very few individual $\mathrm{N}-V$ centers.

To explore the limitations of the pulsed PDMR method, we concentrate on the comparison of the theoretical and experimental values of the noise level. The main components of the total noise $N_{\text {rms }}$ come from the shot noise generated by the photocurrent $I_{\mathrm{SN}}(\mathrm{rms})$ [37,38], input noise of the preamplifier $I_{\text {preamp }}(\mathrm{rms})$, and input noise of the lock-in amplifier $I_{\text {lock-in }}(\mathrm{rms})$ expressed as

$$
N_{\mathrm{rms}}=\left[\delta I_{\mathrm{SN}}(\mathrm{rms})+\delta I_{\text {preamp }}(\mathrm{rms})+\delta I_{\text {lock-in }}(\mathrm{rms})\right] \sqrt{\Delta f},
$$

where $\Delta f$ is the bandwidth of detection electronics. Thanks to the lock-in amplifier detection, one is able to reduce the noise significantly, since in this case, $\Delta f$ corresponds to the equivalent noise bandwidth (ENBW) determined by the lock-in amplifier time constant. The ENBW can be then very narrow, thus, increasing the SNR by rejecting the white noise [37]. The total noise is then calculated as
$N_{\mathrm{rms}}=\left[\sqrt{e \cdot I}+\delta I_{\text {preamp }}(\mathrm{rms})\right]+\delta I_{\text {lock-in }}(\mathrm{rms}) \cdot \sqrt{\mathrm{ENBW}}$,

where $e$ is the elementary charge, and $I$ is the measured photocurrent. Considering the specifications for our preamplifier and lock-in amplifier, we calculate for the CW technique $N_{\text {rms }} / \sqrt{\text { ENBW }}$ of approximately $60.4 \mathrm{fA} \mathrm{Hz}^{-1 / 2}$ with a dominant noise originating from the preamplifier (approximately $60 \mathrm{fA} \mathrm{Hz}^{-1 / 2}$ at a given frequency) compared to the measured value of approximately $83.7 \mathrm{fA} \mathrm{Hz}^{-1 / 2}$ for $1000 \mathrm{~N}-V \mathrm{~s}$ in the focus. We speculate that this difference $\left(23.3 \mathrm{fA} \mathrm{Hz}^{-1 / 2}\right)$ between the experimental and predicted noise is due to the photocurrent noise correlated to MW cross talk occurring on the chip level. This type of cross-talk noise should be significantly reduced for the pulsed measurements where the laser readout pulse is decoupled from the MW pulse. To confirm this, we determine the correlated noise for the experiment in Fig. 5(a) performed using the pulsed protocols. In this configuration, the correlated noise is reduced to $4.4 \mathrm{fA} \mathrm{Hz}^{-1 / 2}$. The correlated noise compared to the optically-triggered $\mathrm{CW}$ PDMR (212.5 $\left.\mathrm{fA} \mathrm{Hz}^{-1 / 2}\right)$ is, therefore, reduced by a factor of 48. The shot noise for the MW-triggered PDMR is approximately $0.126 \mathrm{fA} \mathrm{Hz}^{-1 / 2}$ calculated for a single $\mathrm{N}-V$ spin photocurrent (considering a differential current between the spin $|0\rangle$ and $| \pm 1\rangle$ states of $100 \mathrm{fA}$ ).

\section{CONCLUSION}

To conclude, we demonstrate pulsed coherent readout techniques for small ensembles down to five individual $\mathrm{N}-V$ centers in shallow implanted electronic grade diamond, which is the material used for relevant quantum applications. We develop MW-referenced pulse sequences enabling us to eliminate the $\mathrm{N}_{S}{ }^{0}$-related background photocurrents, which was previously identified as a main obstacle to using the PDMR technique for readout of single $\mathrm{N}-V$ spins. In this way, together with the lock-in readout technique, we reach a signal detection contrast close to $100 \%$. A significant reduction of the correlated noise compared to the $\mathrm{CW}$ technique makes possible an order of magnitude enhancement of the SNR. We develop hybrid Rabi and Ramsey photoelectric coherent spin manipulation protocols encoding high-frequency MW and laser-pulse sequences into a low-frequency envelope. A further enhancement in the SNR can be achieved by designing nanoscopic electrodes for operating efficiently single $\mathrm{N}-V \mathrm{~s}$ with high photoelectron gain $[8,9]$. The ability to read out and control the spin state by the hybrid photocurrent method presented here demonstrates perspectives on progressing towards a compact and scalable single-spin device.

\section{ACKNOWLEDGMENTS}

Support from the EU (FP7 project DIADEMS, Grant No. 611143), Grant Agency of the Czech 
Republic (Project No. 16-16336S), Czech-Flemish bilateral Project No. BOF15BL08, and Flemish Scientific Research Project (FWO) No. G0E7417N are acknowledged. We thank Professor Dmitry Budker from Johannes Gutenberg University, Mainz and from University of California, Berkeley for valuable discussions.

[1] S. Pirandola and S. L. Braunstein, Physics: Unite to build a quantum Internet, Nature (London) 532, 169 (2016).

[2] A. Morello, J. J. Pla, F. A. Zwanenburg, K. W. Chan, K. Y. Tan, H. Huebl, M. Möttönen, C. D. Nugroho, C. Yang, J. A. van Donkelaar, A. D. C. Alves, D. N. Jamieson, C. C. Escott, L. C. L. Hollenberg, R. G. Clark, and A. S. Dzurak, Single-shot readout of an electron spin in silicon, Nature (London) 467, 687 (2010).

[3] G. Balasubramanian, P. Neumann, D. Twitchen, M. Markham, R. Kolesov, N. Mizuochi, J. Isoya, J. Achard, J. Beck, J. Tissler, V. Jacques, P. R. Hemmer, F. Jelezko, and J. Wrachtrup, Ultralong spin coherence time in isotopically engineered diamond, Nat. Mater. 8, 383 (2009).

[4] P. Maletinsky, S. Hong, M. S. Grinolds, B. Hausmann, M. D. Lukin, R. L. Walsworth, M. Loncar, and A. Yacoby, A robust scanning diamond sensor for nanoscale imaging with single nitrogen-vacancy centres, Nat. Nanotechnol. 7, 320 (2012).

[5] P. Neumann, I. Jakobi, F. Dolde, C. Burk, R. Reuter, G. Waldherr, J. Honert, T. Wolf, A. Brunner, and J. H. Shim, High-precision nanoscale temperature sensing using single defects in diamond, Nano Lett. 13, 6 (2013).

[6] T. Staudacher, F. Shi, S. Pezzagna, J. Meijer, J. Du, C. A. Meriles, F. Reinhard, and J. Wrachtrup, Nuclear magnetic resonance spectroscopy on a (5-nanometer $)^{3}$ sample volume, Science 339, 561 (2013).

[7] A. Gruber, A. Dräbenstedt, C. Tietz, L. Fleury, J. Wrachtrup, and C. von Borczyskowski, Scanning confocal optical microscopy and magnetic resonance on single defect centers, Science 276, 2012 (1997).

[8] E. Bourgeois, A. Jarmola, P. Siyushev, M. Gulka, J. Hruby, F. Jelezko, D. Budker, and M. Nesladek, Photoelectric detection of electron spin resonance of nitrogen-vacancy centres in diamond, Nat. Commun. 6, 8577 (2015).

[9] E. Bourgeois, E. Londero, K. Buczak, J. Hruby, M. Gulka, Y. Balasubramaniam, G. Wachter, J. Stursa, K. Dobes, F. Aumayr, M. Trupke, A. Gali, and M. Nesladek, Enhanced photoelectric detection of $\mathrm{NV}$ magnetic resonances in diamond under dual-beam excitation, Phys. Rev. B 95, 041402(R) (2017).

[10] W. F. Koehl, B. B. Buckley, F. J. Heremans, G. Calusine, and D. D. Awschalom, Room temperature coherent control of defect spin qubits in silicon carbide, Nature (London) 479, 84 (2011).

[11] H. Seo, H. M. Govoni, and G. Galli, Design of defect spins in piezoelectric aluminum nitride for solid-state hybrid quantum technologies, Sci. Rep. 6, 20803 (2016).

[12] A. Zrenner, E. Beham, S. Stufler, F. Findeis, M. Bichler, and G. Abstreiter, Coherent properties of a two-level system based on a quantum-dot photodiode, Nature (London) $\mathbf{4 1 8}$, 612 (2002).
[13] Y. Wu, I. M. Piper, M. Ediger, P. Brereton, E. R. Schmidgall, P. R. Eastham, M. Hugues, M. Hopkinson, and R. T. Phillips, Population Inversion in a Single InGaAs Quantum Dot Using the Method of Adiabatic Rapid Passage, Phys. Rev. Lett. 106, 067401 (2011).

[14] F. Hoehne, L. Dreher, M. Suckert, D. P. Franke, M. Stutzmann, and Martin S. Brandt, Time constants of spin-dependent recombination processes, Phys. Rev. B 88, 155301 (2013).

[15] F. Hoehne, L. Dreher, D. P. Franke, M. Stutzmann, L. S. Vlasenko, K. M. Itoh, and M. S. Brandt, Submillisecond Hyperpolarization of Nuclear Spins in Silicon, Phys. Rev. Lett. 114, 117602 (2015).

[16] F. M. Hrubesch, G. Braunbeck, A. Voss, M. Stutzmann, and M.S. Brandt, Broadband electrically detected magnetic resonance using adiabatic pulses, J. Magn. Reson. 254, 62 (2015).

[17] J. Chen, S. Lourette, K. Rezai, T. Hoelzer, M. Lake, M. Nesladek, L.-S. Bouchard, P. Hemmer, and D. Budker, Optical quenching and recovery of photoconductivity in single-crystal diamond, Appl. Phys. Lett. 110, 011108 (2017).

[18] F. M. Hrubesch, G. Braunbeck, M. Stutzmann, F. Reinhard, and M.S. Brandt, Efficient Electrical Spin Readout of $\mathrm{NV}^{-}$Centers in Diamond, Phys. Rev. Lett. 118, 037601 (2017).

[19] M. Mrózek, J. Mlynarczyk, D. S. Rudnicki, and W. Gawlik, Circularly polarized microwaves for magnetic resonance study in the $\mathrm{GHz}$ range: Application to nitrogen-vacancy in diamonds, Appl. Phys. Lett. 107, 013505 (2015).

[20] P. Siyushev, H. Pinto, M. Vörös, A. Gali, F. Jelezko, and J. Wrachtrup, Optically Controlled Switching of the Charge State of a Single Nitrogen-Vacancy Center in Diamond at Cryogenic Temperatures, Phys. Rev. Lett. 110, 167402 (2013).

[21] K. Jensen, V. M. Acosta, A. Jarmola, and D. Budker, Light narrowing of magnetic resonances in ensembles of nitrogenvacancy centers in diamond, Phys. Rev. B 87, 014115 (2013).

[22] See Supplemental Material at http://link.aps.org/ supplemental/10.1103/PhysRevApplied.7.044032 for additional information about methods, materials and data fitting.

[23] G. Balasubramanian, I. Y. Chan, R. Kolesov, M. Al-Hmoud, J. Tisler, C. Shin, C. Kim, A. Wojcik, P. R. Hemmer, A. Krueger, T. Hanke, A. Leitenstorfer, R. Bratschitsch, F. Jelezko, and J. Wrachtrup, Nanoscale imaging magnetometry with diamond spins under ambient conditions, Nature (London) 455, 648 (2008).

[24] F. Jelezko and J. Wrachtrup, Single defect centres in diamond: A review, Phys. Status Solidi A 203, 3207 (2006).

[25] M. W. Doherty, N. B. Manson, P. Delaney, F. Jelezko, J. Wrachtrup, and L. C. L. Hollenberg, The nitrogen-vacancy colour centre in diamond, Phys. Rep. 528, 1 (2013).

[26] L. J. Rogers, M. W. Doherty, M. S. J. Barson, S. Onoda, T. Ohshima, and N.B. Manson, Singlet levels of the $\mathrm{NV}^{-}$centre in diamond, New J. Phys. 17, 013048 (2015).

[27] V. M. Acosta, A. Jarmola, E. Bauch, and D. Budker, Optical properties of the nitrogen-vacancy singlet levels in diamond, Phys. Rev. B 82, 201202(R) (2010).

[28] J.-P. Tetienne, L. Rondin, P. Spinicelli, M. Chipaux, T. Debuisschert, J.-F. Roch, and V. Jacques, Magnetic-fielddependent photodynamics of single NV defects in diamond: 
an application to qualitative all-optical magnetic imaging, New J. Phys. 14, 103033 (2012).

[29] T. Wolf, P. Neumann, K. Nakamura, H. Sumiya, T. Ohshima, J. Isoya, and J. Wrachtrup, Subpicotesla Diamond Magnetometry, Phys. Rev. X 5, 041001 (2015).

[30] R. Hanson, O. Gywat, and D. D. Awschalom, Roomtemperature manipulation and decoherence of a single spin in diamond, Phys. Rev. B 74, 161203(R) (2006).

[31] L. Childress, M. V. Gurudev Dutt, J. M. Taylor, A. S. Zibrov, F. Jelezko, J. Wrachtrup, P. R. Hemmer, and M. D. Lukin, Coherent dynamics of coupled electron and nuclear spin qubits in diamond, Science 314, 281 (2006).

[32] H. Xin, L. Gang-Qin, X. Zhang-Cheng, and P. Xin-Yu, Influence of microwave detuning on Ramsey fringes of a single nitrogen vacancy center spin in diamond, Chin. Phys. Lett. 29, 2 (2012).

[33] C. Grèzes, Towards a Spin-Ensemble Quantum Memory for Superconducting Qubits (Springer International Publishing, New York, 2015).
[34] G.-Q. Liu, X.-Y. Pan, Z.-F. Jiang, N. Zhao, and R.-B. Liu, Controllable effects of quantum fluctuations on spin freeinduction decay at room temperature, Sci. Rep. 2, 432 (2012).

[35] J. M. Taylor, P. Cappellaro, L. Childress, L. Jiang, D. Budker, P. R. Hemmer, A. Yacoby, R. Walsworth, and M. D. Lukin, High-sensitivity diamond magnetometer with nanoscale resolution, Nat. Phys. 4, 810 (2008).

[36] V. R. Horowitz, B. J. Alemán, D. J. Christle, A. N. Cleland, and D. D. Awschalom, Electron spin resonance of nitrogenvacancy centers in optically trapped nanodiamonds, Proc. Natl. Acad. Sci. U.S.A. 109, 13493 (2012).

[37] A. Mandelis, Signal-to-noise ratio in lock-in amplifier synchronous detection: A generalized communications systems approach with applications to frequency, time, and hybrid (rate window) photothermal measurements, Rev. Sci. Instrum. 65, 3309 (1994).

[38] R. Pettai, Noise in Receiving Systems (Wiley-Interscience, New York, 1984). 